\title{
Better Approximation Algorithms for Grasp-and-Delivery Robot Routing Problems
}

\author{
Aleksandar SHURBEVSKI ${ }^{\dagger}$, Nonmember, Hiroshi NAGAMOCHI ${ }^{\dagger \dagger a}$, Member, $^{\dagger}$ \\ and Yoshiyuki KARUNO ${ }^{\dagger \dagger}$, Nonmember
}

\begin{abstract}
SUMMARY In this paper, we consider a problem of simultaneously optimizing a sequence of graphs and a route which exhaustively visits the vertices from each pair of successive graphs in the sequence. This type of problem arises from repetitive routing of grasp-and-delivery robots used in the production of printed circuit boards. The problem is formulated as follows. We are given a metric graph $G^{*}=\left(V^{*}, E^{*}\right)$, a set of $m+1$ disjoint subsets $C_{i} \subseteq V^{*}$ of vertices with $\left|C_{i}\right|=n, i=0,1, \ldots, m$, and a starting vertex $s \in C_{0}$. We seek to find a sequence $\pi=\left(C_{i_{1}}, C_{i_{2}}, \ldots, C_{i_{m}}\right)$ of the subsets of vertices and a shortest walk $P$ which visits all $(m+1) n$ vertices in $G^{*}$ in such a way that after starting from $s$, the walk alternately visits the vertices in $C_{i_{k-1}}$ and $C_{i_{k}}$, for $k=1,2, \ldots, m\left(i_{0}=0\right)$. Thus, $P$ is a walk with $m(2 n-1)$ edges obtained by concatenating $m$ alternating Hamiltonian paths between $C_{i_{k-1}}$ and $C_{i_{k}}, k=1,2, \ldots, m$. In this paper, we show that an approximate sequence of subsets of vertices and an approximate walk with at most three times the optimal route length can be found in polynomial time.

key words: combinatorial optimization, routing and scheduling, industrial robots, approximation algorithms, metric traveling salesperson problem
\end{abstract}

\section{Introduction}

This research subject was motivated by a routing problem of a grasp-and-delivery robot used at an actual printed circuit board assembly line. The assembly line can process at most one printed circuit board at a time. A new (unprocessed) printed circuit board is brought into the assembly line along an automatic guided railway, and is released from there by the railway immediately after it is finished.

Let $\mathcal{J}=\left\{J_{1}, J_{2}, \ldots, J_{m}\right\}$ denote a set of $m$ printed circuit boards to be processed at the assembly line. In practice, processing of a printed circuit board involves an automated manipulator embedding electronic parts in the board from above. (In this paper, we do not treat any automated manipulator routing issue, e.g., see Srivastav, Schroeter and Michel [15].) As a printed circuit board is processed, $n$ identical pins support the printed circuit board from underneath to prevent it from overbending. The board size is common

Manuscript received March 28, 2012.

Manuscript revised July 27, 2012.

†The author is with the Doctoral Program, Department of Applied Mathematics and Physics, Graduate School of Informatics, Kyoto University, Kyoto-shi, 606-8501 Japan.

${ }^{\dagger}$ The author is with the Department of Applied Mathematics and Physics, Graduate School of Informatics, Kyoto University, Kyoto-shi, 606-8501 Japan.

${ }^{\dagger \dagger}$ The author is with the Department of Mechanical and System Engineering, Graduate School of Science and Technology, Kyoto Institute of Technology, Kyoto-shi, 606-8585 Japan.

a) E-mail: nag@amp.i.kyoto-u.ac.jp

DOI: 10.1587/transinf.E96.D.450 for all $m$ printed circuit boards, and pins are scattered in a given bounded two-dimensional area. The distances between possible locations of the pins are Euclidean.

Each printed circuit board has its own pattern of circuit and a dedicated pin configuration, $C_{i}$, is designed for every printed circuit board $J_{i}$ so that the pins do not obstruct the circuit. This implies that if a printed circuit board $J_{i}$ is followed by another $J_{j}$ in a processing order of $m$ printed circuit boards, the current pin configuration $C_{i}$ needs to be changed to $C_{j}$ during the time interval between releasing $J_{i}$ and sending for $J_{j}$. The assembly line employs a single grasp-and-delivery robot to change pin configurations.

The grasp-and-delivery robot can grasp at most one pin at a time by using its end-effector, i.e., it is a material handling device with unit capacity, not unlike a human hand. After grasping a pin, the robot delivers it from its current location to another one in the given bounded area. In this paper, we assume that the travel lengths of the grasp-anddelivery robot between possible locations of pins are symmetric and satisfy the triangle inequality, since the distances between possible pin locations in the given bounded area are Euclidean.

Each pin configuration $C_{i}, i=1,2, \ldots, m$, can also be regarded as a subset of vertices of a metric graph $G^{*}=$ $\left(V^{*}, E^{*}\right)$. Let $\pi=\left(C_{i_{1}}, C_{i_{2}}, \ldots, C_{i_{m}}\right)$ denote a graph sequence, which is a permutation on the given $m$ subsets of vertices. Also it can represent a processing order of $m$ printed circuit boards. For each $k=1,2, \ldots, m$, the grasp-and-delivery robot arranges $n$ pins from pin configuration $C_{i_{k-1}}$ to the immediately following $C_{i_{k}}$ by transferring the pins one by one. We call such an arrangement from a pin configuration to another one a transition. For notational convenience, let $C_{0}$ denote a given initial pin configuration, hence $i_{0}=0$. A transfer route of the grasp-and-delivery robot is a walk in the metric graph $G^{*}$. Particularly, a walk corresponding to a transfer route is a multiset of vertices, but strictly a set of edges in $G^{*}$ (i.e., vertices may be repeated, while edges are not).

The repetitive routing problem to be discussed in this paper asks to find a graph sequence and a walk of the graspand-delivery robot so that the route length over all $m$ transitions is minimized. The pin to be grasped first in the initial pin configuration $C_{0}$ is prescribed.

Note that the current configuration $C_{i_{k-1}}$ in the $k$-th transition has been the next required one in the preceding, $(k-1)$-th transition. In every transition except for the first 
one, the grasp-and-delivery robot starts by grasping the pin transferred last in the preceding transition, since it is not advantageous for the grasp-and-delivery robot to start by grasping another pin. We regard this condition as a constraint of consecutiveness.

In a somewhat restricted form, without considering a processing order of printed circuit boards, the repetitive routing problem has been described by Karuno, Nagamochi and Shurbevski [9], where they have shown it can be approximated within factor two in polynomial time. The problem of simultaneously determining a graph sequence as well as a walk of the grasp-and-delivery robot has been addressed by the same authors in [10] and [11], where they have also provided polynomial-time approximation algorithms with approximation factors of eight (with $O\left(m^{2} n^{3}\right)$ time complexity) and six (with $O\left(m^{3}+m^{2} n^{3}\right)$ time complexity), respectively. More detailed revision and statement of the repetitive routing problem are given in Sect. 2 .

In this paper, we show that an approximate graph sequence and an approximate walk with route length at most three times the optimum, can be found in polynomial time. We first construct a universal graph, by adding a node to represent each subset $C_{i}$ of vertices. The universal graph is complete and there is an arc between every two nodes. Then we define a cost for each arc in the universal graph, and further define a metric in the universal graph based on these costs. By using Hoogeveen's modification [7] of Christofides' 3/2-approximation [2] for the metric traveling salesperson problem, we obtain an approximate graph sequence. In order to derive the constant factor performance guarantee, we use a minimum weight alternating spanning tree in a weighted complete bipartite graph to define the cost function in the universal graph. The minimum weight alternating spanning tree can be obtained by applying a matroid intersection algorithm presented by Frank [4].

\section{Problem Description}

For each $i=0,1, \ldots, m$, let $C_{i}$ be a subset of vertices with $\left|C_{i}\right|=n$ corresponding to the pin configuration required for printed circuit board $J_{i}$, where $J_{0}$ is a fictitious board, serving only to define a given initial pin configuration $C_{0}$. Since the pin to be grasped first in the initial configuration is prescribed, let $s \in C_{0}$ denote the corresponding starting vertex of the grasp-and-delivery robot. Any two distinct subsets $C_{i}$ and $C_{j}(i \neq j)$ of vertices are disjoint from each other.

Let $G^{*}=\left(V^{*}, E^{*}\right)$ be a graph, where $V^{*}=\bigcup_{i=0}^{m} C_{i}$ is the set of $(m+1) n$ vertices defined by the union of $m+1$ subsets of vertices, and $E^{*}=V^{*} \times V^{*}$ is the set of edges. Let $u \in V^{*}$ and $v(\neq u) \in V^{*}$ be two end vertices of an edge $e \in E^{*}$, i.e., $e=\{u, v\}$, and $w(e)=w(u, v)$ be a weight function. We assume that the weight function $w$ is symmetric, i.e., $w(u, v)=w(v, u)$ for an edge $e=\{u, v\} \in E^{*}$, and satisfies the triangle inequality. We may extend the notation $w$, so that for $G \subseteq G^{*}, w(G)$ denotes $\sum_{e \in G} w(e)$. Likewise, for a walk $P$ in $G^{*}, w(P)=\sum_{e \in P} w(e)$.

For each pair of distinct subsets $C_{i}$ and $C_{j}$ of ver- tices, we define a complete bipartite graph $B_{i, j}=\left(V_{i, j}=\right.$ $C_{i} \cup C_{j}, E_{i, j}=C_{i} \times C_{j}$ ) with edge weight function $w$, where $V_{i, j}$ is the set of $2 n$ vertices and $E_{i, j}$ is the set of $n^{2}$ edges. For a complete bipartite graph $B_{i, j}$, let $P_{i, j}$ denote an alternating Hamiltonian path, where the vertices in $C_{i}$ and $C_{j}$ appear alternately. Similarly, let $H_{i, j}$ denote an alternating Hamiltonian cycle in $B_{i, j}$. Note that an alternating Hamiltonian path $P_{i, j}$ contains $2 n-1$ edges, while an alternating Hamiltonian cycle $H_{i, j}$ contains $2 n$ edges.

A graph sequence $\pi=\left(C_{i_{1}}, C_{i_{2}}, \ldots, C_{i_{m}}\right)$ is a permutation of $m$ subsets $C_{1}, C_{2}, \ldots, C_{m}$ of vertices. A fixed graph sequence may be denoted by $\pi=\left(C_{1}, C_{2}, \ldots, C_{m}\right)$ for notational simplification. Given a fixed graph sequence $\pi$, finding an optimal transfer route to visit the vertices in $G^{*}$ is described as the following problem:

Problem FRR (Fixed sequence Repetitive Routing)

INSTANCE: A metric graph $G^{*}=\left(V^{*}, E^{*}\right)$ with weight function $w$, a set of $m+1$ disjoint subsets $C_{i} \subseteq V^{*}$ of vertices with $\left|C_{i}\right|=n, i=0,1, \ldots, m$, a starting vertex $s \in C_{0}$, and a fixed graph sequence $\pi=\left(C_{1}, C_{2}, \ldots, C_{m}\right)$.

FEASIBLE SOLUTION: A repetitive walk $P$ which visits all vertices in $G^{*}$ in such a way that after starting from $s \in$ $C_{0}$, it alternately visits all the vertices in $C_{i-1}$ and $C_{i}$ for $i=1,2, \ldots, m$.

GOAL: Find a shortest repetitive walk $P=P^{*}$ which minimizes the route length $w(P)$ with respect to $\pi$.

Note that $P$ is a walk with $m(2 n-1)$ edges obtained by concatenating $m$ alternating Hamiltonian paths $P_{i-1, i}$ between $C_{i-1}$ and $C_{i}, i=1,2, \ldots, m$. Problem FRR is known to be NP-hard (see Garey and Johnson [6], Krishnamoorthy [13]). Karuno, Nagamochi and Shurbevski have shown that problem FRR admits a polynomial time approximation algorithm with factor two [9].

In problem FRR, we are not concerned with the production order of the printed circuit boards, i.e., the graph sequence, and are not perplexed by an inversion in the order of $C_{i-1}$ and $C_{i}$ for the trailing $m-1$ transitions, $i=$ $2,3, \ldots, m$ (we must start from $C_{0}$ however). In this paper, we also consider the choice of a graph sequence among all $m$ ! possible graph sequences of the $m$ subsets of vertices, $\left\{C_{1}, C_{2}, \ldots, C_{m}\right\}$, to find a shortest repetitive walk which visits all vertices in $G^{*}$. More formally, this flavor of the repetitive routing problem is formulated as follows.

Problem PRR (Permutable sequence Repetitive Routing) INSTANCE: A metric graph $G^{*}=\left(V^{*}, E^{*}\right)$ with weight function $w$, a set of $m+1$ disjoint subsets $C_{i} \subseteq V^{*}$ of vertices with $\left|C_{i}\right|=n, i=0,1, \ldots, m$, and a starting vertex $s \in C_{0}$. FEASIBLE SOLUTION: A repetitive walk $P$ which visits all vertices in $G^{*}$ in such a way that after starting from $s \in C_{0}$, following a resulting graph sequence $\pi=$ $\left(C_{i_{1}}, C_{i_{2}}, \ldots, C_{i_{m}}\right)$, it visits the vertices in $C_{i_{k-1}}$ and $C_{i_{k}}$ alternately for $k=1,2, \ldots, m$.

GOAL: Find a shortest repetitive walk $P=P^{*}$ which minimizes the route length $w(P)$.

In this paper, we show that problem PRR admits a 
polynomial time approximation algorithm with factor three, which improves the previously known factors, eight [10] and six [11]. The key to this significant improvement is to overcome the difficulty arising from an inversion in the order of any two subsets $C_{i}$ and $C_{j}$ in the graph sequence. This is difficult due to the special structure of an alternating spanning tree on the vertices of the subsets $C_{i}$ and $C_{j}$.

To achieve the factor three, we introduce new ideas in constructing the universal graph $\mathcal{G}$ as a complete graph that spans all subsets $C_{i}$ of vertices as its nodes. Each arc joining two distinct nodes $C_{i}$ and $C_{j}$ is assigned a cost, which is itself a lower bound on the length of an alternating Hamiltonian path $P_{i, j}$ between $C_{i}$ and $C_{j}$. The costs are used to define a transition distance function which is a metric in the universal graph. To determine an approximate graph sequence $\pi^{\prime}$, we compute a 3/2-approximate Hamiltonian path in the metric closure of $\mathcal{G}$ [2], [7]. Based on the approximate graph sequence, we obtain an approximate repetitive walk $P^{\prime}$ which visits all vertices in $G^{*}$. At this stage, we use a nice property of alternating Hamiltonian cycles that, given two alternating Hamiltonian cycles $H_{i, j}$ and $H_{j, k}$, there exists an alternating Hamiltonian cycle $H_{i, k}$ with $w\left(H_{i, k}\right) \leq w\left(H_{i, j}\right)+w\left(H_{j, k}\right)$. This triangle inequality allows us to construct a 2-approximate repetitive walk with respect to the approximate graph sequence $\pi^{\prime}$. Moreover, the approximation algorithm with factor two is a more sophisticated version of the one presented by Karuno, Nagamochi and Shurbevski [9]. Similarly to [9] though, alternating spanning trees are also used to obtain the alternating Hamiltonian cycles.

The combination of the two approximations, a graph sequence and a repetitive walk with respect to it, yields a 3-approximate solution to problem PRR. In the following sections, we explain this approximation technique in further detail.

\section{Constructing the Universal Graph}

In this section, we construct the universal graph $\mathcal{G}=(C=$ $\left\{C_{i} \mid i=0,1, \ldots, m\right\}, \mathcal{E}=C \times C$ ) by representing each subset $C_{i}$ of vertices as a node and adding an arc $a=\left\{C_{i}, C_{j}\right\}$ between every two nodes $C_{i}$ and $C_{j}$ in $C$. In order to make a distinction and simplify exposition, we respectively use the terms node and arc for a vertex and an edge in the universal graph. The graph $\mathcal{G}$ is used to determine an approximate graph sequence $\pi^{\prime}=\left(C_{i_{1}}, C_{i_{2}}, \ldots, C_{i_{m}}\right)$.

We first explain how to define a cost function $\omega$ for $\operatorname{arcs}$ in $\mathcal{G}$. Given a weighted complete bipartite graph $B_{i, j}=$ $\left(V_{i, j}=C_{i} \cup C_{j}, E_{i, j}=C_{i} \times C_{j}\right)$, a spanning tree $T_{i, j}$ in $B_{i, j}$ is called an alternating spanning tree (with respect to $C_{i}$ ) if the degree of any vertex of $C_{i}$ is at most two (see Fig. 1).

The following lemma concerning alternating spanning trees has been demonstrated (see Baltz and Srivastav [1]).

Lemma 1: [1] Let $B_{i, j}=\left(V_{i, j}=C_{i} \cup C_{j}, E_{i, j}=C_{i} \times C_{j}\right)$ be a complete bipartite graph in $G^{*}$, and let $T_{i, j}$ be an alternating spanning tree in $B_{i, j}$. Then, there is exactly one vertex of $C_{i}$ whose degree in $T_{i, j}$ is one.

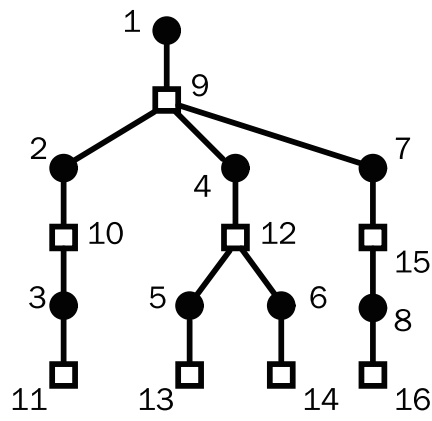

Fig. 1 An alternating spanning tree $T_{0,1}$ with $n=8$, where the degree of each black vertex $\left(\in C_{0}\right)$ is at most two.

An alternating spanning tree $T_{i, j}^{*}$ is called a minimum weight alternating spanning tree if it satisfies $w\left(T_{i, j}^{*}\right) \leq$ $w\left(T_{i, j}\right)$ for any alternating spanning tree $T_{i, j}$ in $B_{i, j}$ with weight function $w$.

Lemma 2: [1] Let $B_{i, j}=\left(V_{i, j}=C_{i} \cup C_{j}, E_{i, j}=C_{i} \times C_{j}\right)$ be a weighted complete bipartite graph, induced by $C_{i} \cup C_{j}$ in $G^{*}$. Then, a minimum weight alternating spanning tree $T_{i, j}^{*}$ can be computed in $O\left(n^{7}\right)$ time.

The above lemma can be obtained following a matroid intersection algorithm (see Frank [4] and the textbook by Korte and Vygen [12]). The time complexity follows Baltz and Srivastav [1].

Let $\tilde{P}_{i, j}$ be an alternating Hamiltonian path (with respect to $C_{i}$ ) in $B_{i, j}$ which minimizes the path length among all choices of starting and end vertices. We call $\tilde{P}_{i, j}$ a minimum weight alternating Hamiltonian path in $B_{i, j}$. By noting that any alternating Hamiltonian path $P_{i, j}$ is also an alternating spanning tree in $B_{i, j}$, and $w\left(\tilde{P}_{i, j}\right)=w\left(\tilde{P}_{j, i}\right)$, we have the following lemma.

Lemma 3: Let $\tilde{P}_{i, j}$ be a minimum weight alternating Hamiltonian path in $B_{i, j}$. Then, it holds

$$
w\left(\tilde{P}_{i, j}\right) \geq \max \left\{w\left(T_{i, j}^{*}\right), w\left(T_{j, i}^{*}\right)\right\} .
$$

Using the minimum weight $w\left(T_{i, j}^{*}\right)$ of an alternating spanning tree, we set the cost $\omega\left(C_{i}, C_{j}\right)$ of an arc $a=\left\{C_{i}, C_{j}\right\}$ in $\mathcal{G}$ to be

$$
\omega\left(C_{i}, C_{j}\right)=\min \left\{w\left(T_{i, j}^{*}\right), w\left(T_{j, i}^{*}\right)\right\} .
$$

Next, we define the transition distance between two nodes $C_{i}$ and $C_{j}$, denoted by $d\left(C_{i}, C_{j}\right)$. Namely, $d\left(C_{i}, C_{j}\right)$ is the length of a shortest path between the two nodes $C_{i}$ and $C_{j}$ in $\mathcal{G}$ with respect to the cost function $\omega$. Obviously, the transition distance function $d$ is symmetric and satisfies the triangle inequality. To formalize the transition distance function $d$ as a metric in $\mathcal{G}$, we define $d\left(C_{i}, C_{j}\right)=0$ for $i=j$. As with $w$, for a subgraph $\mathcal{G}^{\prime}$ of $\mathcal{G}, d\left(\mathcal{G}^{\prime}\right)$ denotes $\sum_{a \in \mathcal{G}^{\prime}} d(a)$. From Eqs. (1) and (2), we have

$$
w\left(\tilde{P}_{i, j}\right) \geq \omega\left(C_{i}, C_{j}\right) \geq d\left(C_{i}, C_{j}\right) .
$$

Let $H_{i, j}^{*}$ be an alternating Hamiltonian cycle in $B_{i, j}$ 
which minimizes the cycle length in $B_{i, j}$. We call $H_{i, j}^{*}$ a minimum weight alternating Hamiltonian cycle. By traversing a minimum weight alternating spanning tree $T_{i, j}^{*}$ starting from the vertex in $C_{i}$ with degree one (see Lemma 1) in a depth-first manner with shortcuts, we can obtain an alternating Hamiltonian cycle $H_{i, j}^{\prime}$ in $B_{i, j}$ such that it satisfies the following lemma (since the weight function $w$ in $G^{*}$ satisfies the triangle inequality).

Lemma 4: Let $H_{i, j}^{*}$ be a minimum weight alternating Hamiltonian cycle in $B_{i, j}$ and let $H_{i, j}^{\prime}$ be an alternating Hamiltonian cycle obtained by traversing either minimum weight alternating spanning tree $T_{i, j}^{*}$ or $T_{j, i}^{*}$. Then, it holds

$w\left(H_{i, j}^{*}\right) \leq w\left(H_{i, j}^{\prime}\right) \leq 2 \omega\left(C_{i}, C_{j}\right)$.

Before proceeding, we briefly digress by presenting a convenient property which holds for simple Eulerian graphs. Consider a simple Eulerian graph $G=(V, E)$, with a distinguished vertex $v \in V$ of degree four (having exactly four neighbors, different from each other). There are three different ways to split the vertex $v$ and eliminate it from $G$, namely replacing the four edges incident to it by two new edges (each edge shortcuts two of the original edges incident with $v$ ) connecting two pairs of the four distinct neighbors. By removing $v$ with this procedure, the degrees of the vertices in $V-\{v\}$ remain unchanged.

Lemma 5: Let $G=(V, E)$ be a simple Eulerian graph, with a distinguished vertex $v \in V$ of degree four. Among the three different ways to split $v$ by replacing two pairs of edges incident to it in a shortcutting manner, there are at least two ways in which the graph obtained by splitting $v$ remains connected and thus Eulerian.

Proof. Let $G^{\prime}$ denote one of the three possible resulting graphs obtained as described above. Assume that $G^{\prime}$ is disconnected. Then, $G^{\prime}$ has two components $G_{1}^{\prime}$ and $G_{2}^{\prime}$. We see that $G_{h}^{\prime}(h=1,2)$ must remain connected even if we remove the newly formed edges, since otherwise there would be components which would have only one odd degree vertex, a contradiction to the fact that the number of vertices with odd degree in a graph is even. Therefore the remaining two ways to split the vertex $v$ must result in a connected graph, for they will connect the odd degree vertices of $G_{1}^{\prime}$ to those of $G_{2}^{\prime}$. Moreover, since the splitting of $v$ does not change the degree of any other vertex, both of them remain Eulerian.

Lemma 6: Let $H_{i, j}^{\prime}$ and $H_{j, k}^{\prime}$ be alternating Hamiltonian cycles in the weighted complete bipartite graphs $B_{i, j}$ and $B_{j, k}$, respectively. Then, an alternating Hamiltonian cycle $H_{i, k}$ in the weighted complete bipartite graph $B_{i, k}$ such that

$$
w\left(H_{i, k}\right) \leq w\left(H_{i, j}^{\prime}\right)+w\left(H_{j, k}^{\prime}\right)
$$

can be constructed from $H_{i, j}^{\prime}$ and $H_{j, k}^{\prime}$ in $O\left(n^{2}\right)$ time.

Proof. Consider the graph $G=\left(C_{i} \cup C_{j} \cup C_{k}, H_{i, j}^{\prime} \cup H_{j, k}^{\prime}\right)$ obtained by joining $H_{i, j}^{\prime}$ and $H_{j, k}^{\prime}$. Note that $G$ is simple, Eulerian, connected and every vertex in $C_{j}$ is of degree four in $G$.

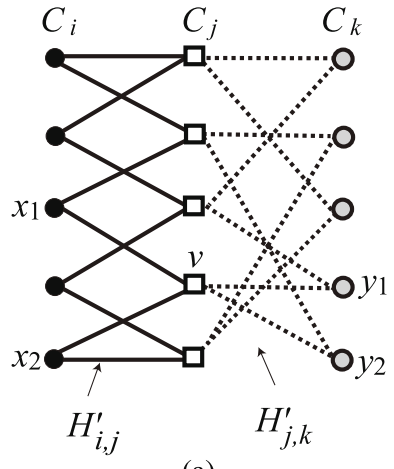

(a)

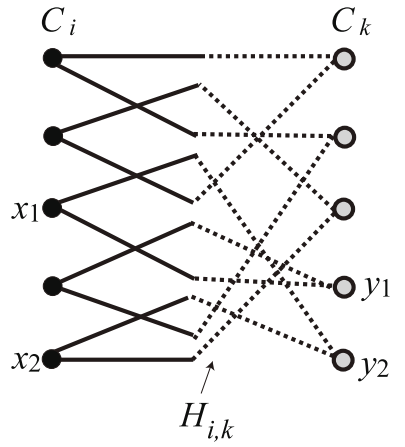

(b)
Fig. 2 (a) Alternating Hamiltonian cycles $H_{i, j}^{\prime}$ and $H_{j, k}^{\prime}$ in $B_{i, j}$ and $B_{j, k}$; (b) An alternating Hamiltonian cycle $H_{i, k}$ in $B_{i, k}$ such that $w\left(H_{i, k}\right) \leq$ $w\left(H_{i, j}^{\prime}\right)+w\left(H_{j, k}^{\prime}\right)$.

Let $v$ be a vertex in $C_{j}$ (as in the proposition of Lemma 5) and $\left\{x_{1}, v\right\},\left\{x_{2}, v\right\},\left\{v, y_{1}\right\}$ and $\left\{v, y_{2}\right\}$ be the four edges incident to $v$ where $x_{1}, x_{2} \in C_{i}$ and $y_{1}, y_{2} \in C_{j}$ (see Fig. 2). Out of the possible ways to split $v \in C_{j}$, we observe the two cases which do not replace the edges $\left\{x_{1}, v\right\}$ and $\left\{x_{2}, v\right\}$ by $\left\{x_{1}, x_{2}\right\}\left(\left\{y_{1}, v\right\}\right.$ and $\left\{y_{2}, v\right\}$ by $\left\{y_{1}, y_{2}\right\}$, respectively). That is, we are only interested in replacing existing edges in such a way that newly added edges have one endpoint in $C_{i}$ and another in $C_{k}$.

Following Lemma 5, we see that the vertex $v$ can be removed by replacing the edges incident to it while preserving connectivity of at least one of the possible resulting graphs. Since each of the vertices in $C_{i} \cup C_{k}$ is of degree two, connectedness implies simplicity. Following, the obtained graph in turn satisfies the proposition of Lemma 5. Hence the same feature can be applied recursively, until all vertices of degree four from the original $C_{j}$ are removed. Notice that due to the triangle inequality (e.g. $w\left(x_{1}, y_{1}\right) \leq$ $\left.w\left(x_{1}, v\right)+w\left(v, y_{1}\right)\right)$ the total weight of any of $G^{\prime}$ and $G^{\prime \prime}$ never exceeds that of $G$. The resulting graph, say $H_{i, k}$, is an alternating Hamiltonian cycle on $C_{i}$ and $C_{k}$. Its length $w\left(H_{i, k}\right)$ is at most that of $G$, i.e., $w\left(H_{i, k}\right) \leq w\left(H_{i, j}^{\prime}\right)+w\left(H_{j, k}^{\prime}\right)$.

The time complexity follows straightforwardly from the observation that the number of steps required in order to check the connectedness of either $G^{\prime}$ or $G^{\prime \prime}$ when removing a vertex from $C_{j}$ is $O(n)$.

Lemma 7: For any pair of distinct subsets $C_{i}$ and $C_{j}$ of vertices, an alternating Hamiltonian cycle $H_{i, j}^{\prime \prime}$ in the weighted complete bipartite graph $B_{i, j}$ such that

$$
w\left(H_{i, j}^{\prime \prime}\right) \leq 2 d\left(C_{i}, C_{j}\right)
$$

can be obtained in $O\left(m n^{2}\right)$ time.

Proof. Without loss of generality, let $\left(C_{i}, C_{i+1}, \ldots, C_{j}\right)$ denote a shortest path between $C_{i}$ and $C_{j}$ in $\mathcal{G}$ with respect to the transition distance function $d$ by re-indexing the nodes if necessary. By definition, it holds $\sum_{i \leq k<j} \omega\left(C_{k}, C_{k+1}\right)=$ $d\left(C_{i}, C_{j}\right)$. For each $i \leq k<j$, let $H_{k, k+1}^{\prime}$ be an alternating Hamiltonian cycle in $B_{k, k+1}$ such that $w\left(H_{k, k+1}^{\prime}\right) \leq$ $2 \omega\left(C_{k}, C_{k+1}\right)$ (see Lemma 4). By applying Lemma 6, we 
have an alternating Hamiltonian cycle $H_{i, j}^{\prime \prime}$ such that

$$
\begin{aligned}
w\left(H_{i, j}^{\prime \prime}\right) & \leq \sum_{i \leq k<j} w\left(H_{k, k+1}^{\prime}\right) \leq 2 \sum_{i \leq k<j} \omega\left(C_{k}, C_{k+1}\right) \\
& =2 d\left(C_{i}, C_{j}\right) .
\end{aligned}
$$

The time complexity is straightforwardly evaluated by Lemma 6.

\section{Finding a Hamiltonian Path in the Universal Graph}

Let $\mathcal{F}^{*}$ denote a shortest (with respect to the transition distance function $d$ ) Hamiltonian path starting from the initial node $C_{0}$ in the universal graph $\mathcal{G}$ and let $d\left(\mathcal{F}^{*}\right)$ denote the sum of transition distances of two consecutive nodes belonging to $\mathcal{F}^{*}$. The problem of finding such a shortest Hamiltonian path in $\mathcal{G}$ is NP-hard (e.g., see Garey and Johnson [6]).

Without loss of generality, let $\pi^{*}=\left(C_{1}, C_{2}, \ldots, C_{m}\right)$ be a graph sequence of $m$ subsets of vertices extracted from a shortest repetitive walk $P^{*}$ for a given instance of problem PRR by re-indexing $m$ printed circuit boards. According to this, let $P^{*}=\left(P_{0,1}^{*}, P_{1,2}^{*}, \ldots, P_{m-1, m}^{*}\right)$ denote the shortest repetitive walk, where $P_{i-1, i}^{*}$ is the $i$-th alternating Hamiltonian path in the weighted complete bipartite graph $B_{i-1, i}$. Then, by definition it holds $w\left(P_{i, j}^{*}\right) \geq w\left(\tilde{P}_{i, j}\right)$.

Lemma 8: For an instance of problem PRR, let $P^{*}$ be a shortest repetitive walk in $G^{*}$. Then, the route length satisfies

$$
w\left(P^{*}\right) \geq d\left(\mathcal{F}^{*}\right) .
$$

Proof. By using Eq. (3), we have

$$
\begin{aligned}
& w\left(P^{*}\right)=\sum_{i=1}^{m} w\left(P_{i-1, i}^{*}\right) \\
& \geq \sum_{i=1}^{m} w\left(\tilde{P}_{i-1, i}\right) \geq \sum_{i=1}^{m} d\left(C_{i-1}, C_{i}\right) \geq d\left(\mathcal{F}^{*}\right) .
\end{aligned}
$$

Christofides' algorithm for the metric traveling salesperson problem delivers a tour whose length is less than $3 / 2$ times the optimum [2]. For the problem of finding a shortest Hamiltonian path starting from a prescribed starting node, a modification of Christofides' algorithm has been presented by Hoogeveen [7], which delivers a Hamiltonian path whose length is no more than $3 / 2$ times the length of an optimal Hamiltonian path (see also Johnson and Papadimitriou [8]). The algorithm is sketched as follows:

(1) Find a minimum weight spanning tree $\mathcal{T}^{*}$ of the universal graph $\mathcal{G}$ with respect to transition distance function $d$ (with weight $d\left(\mathcal{T}^{*}\right)$ );

(2) Construct the set $S_{\text {odd }}$ of all odd-degree nodes in $\mathcal{T}^{*}$;

(3) If the node $C_{0}$ is an odd-degree node in $\mathcal{T}^{*}$, then remove it from the set $S_{\text {odd }}$. Otherwise, add it to the set $S_{\text {odd }}$. We denote the resulting set of nodes by $S$.

(4) After augmenting $S$ with a dummy node, $v_{d}$, such that $d\left(v_{d}, u\right)=0, \forall u \in S$, compute a minimum weight perfect matching $\mathcal{M}^{*}$ on the set $S$ with respect to the distance $d$. (The matching $\mathcal{M}^{*}$ has weight $d\left(\mathcal{M}^{*}\right)$ excluding the arc incident to the dummy node.);

(5) For the subgraph of $\mathcal{G}$ with arcs of $\mathcal{T}^{*}$ and $\mathcal{M}^{*}$, if the node $C_{0}$ belongs to $S$ and it is not incident with any arc of $\mathcal{M}^{*}$, then delete an arbitrary arc which is incident to the node;

(6) For the resulting graph (notice that the graph has two odd-degree nodes, and one of them is the node $C_{0}$ ), find an Eulerian path starting from the node $C_{0}$;

(7) Convert the Eulerian path into a Hamiltonian path $\mathcal{F}^{\prime}$ by applying shortcuts.

Regarding the spanning tree $\mathcal{T}^{*}$ and the matching $\mathcal{M}^{*}$, Hoogeveen [7] showed that

$$
\begin{aligned}
d\left(\mathcal{T}^{*}\right) & \leq d\left(\mathcal{F}^{*}\right), \\
d\left(\mathcal{M}^{*}\right) & \leq \frac{1}{2} d\left(\mathcal{F}^{*}\right) .
\end{aligned}
$$

Since the transition distance function $d$ is a metric in $\mathcal{G}$, by applying the algorithm of Hoogeveen's modification, we obtain the following lemma concerning a Hamiltonian path $\mathcal{F}^{\prime}$ in $\mathcal{G}$ starting from the node $C_{0}$ :

Lemma 9: [7]

$$
\frac{d\left(\mathcal{F}^{\prime}\right)}{d\left(\mathcal{F}^{*}\right)} \leq \frac{3}{2} \text {. }
$$

Computing a minimum weight spanning tree $\mathcal{T}^{*}$ in $\mathcal{G}$ with $O(\mathrm{~m})$ nodes requires $O\left(\mathrm{~m}^{2}\right)$ time (see Prim's algorithm with Fibonacci heaps [5], [14]). It takes $O\left(\mathrm{~m}^{3}\right)$ time by a generalized algorithm of Edmonds [3] to compute a minimum weight matching $\mathcal{M}^{*}$. Since the resulting graph in (6) has $O(m)$ nodes and $O(m)$ arcs, an Eulerian path from the node $C_{0}$ can be found in $O(m)$ time (e.g., see Korte and Vygen [12]).

\section{Performance Guarantee}

The proposed approximation algorithm is summarized as follows. For a given instance of problem PRR, in every pair of distinct subsets $C_{i}$ and $C_{j}$ of vertices, we first compute the minimum weight alternating spanning trees $T_{i, j}^{*}$ and $T_{j, i}^{*}$ in the weighted complete bipartite graph $B_{i, j}=$ $\left(C_{i} \cup C_{j}, C_{i} \times C_{j}\right)\left(=B_{j, i}\right)$.

Next, in every pair of distinct subsets $C_{i}$ and $C_{j}$ of vertices, we obtain alternating Hamiltonian cycles $H_{i, j}^{\prime}$ and $H_{j, i}^{\prime}$ by traversing either $T_{i, j}^{*}$ or $T_{i, j}^{*}$ in a depth-first manner with shortcuts (see Lemma 4).

Then, we construct the universal graph $\mathcal{G}=(C, \mathcal{E}=$ $C \times C$ ) with cost function $\omega$ (see Eq. (2)). From $\omega$, for every pair of distinct nodes $C_{i}$ and $C_{j}$, we calculate the transition distance $d\left(C_{i}, C_{j}\right)$ by finding a shortest path between the two nodes in $\mathcal{G}$ with respect to $\omega$ (see Eq. (3)).

After calculating the transition distances $d$, we find a Hamiltonian path $\mathcal{F}^{\prime}$ in $\mathcal{G}$ whose length satisfies $d\left(\mathcal{F}^{\prime}\right) \leq$ 
$3 w\left(P^{*}\right) / 2$ (see Eqs. (7) and (10)).

Without loss of generality, let $\pi^{\prime}=\left(C_{1}, C_{2}, \ldots, C_{m}\right)$ denote a graph sequence of $m$ subsets of vertices corresponding to $\mathcal{F}^{\prime}$ in $\mathcal{G}$. Let $P^{\prime}=\left(P_{0,1}^{\prime}, P_{1,2}^{\prime}, \ldots, P_{m-1, m}^{\prime}\right)$ be a repetitive walk in $G^{*}$ with respect to $\pi^{\prime}$. Each alternating Hamiltonian path $P_{i-1, i}^{\prime}$ is obtained in the following manner. For each weighted complete bipartite graph $B_{i-1, i}$, $i=1,2, \ldots, m$, we compute an alternating Hamiltonian cycle $H_{i-1, i}^{\prime \prime}$ according to Lemma 7 (see Eq. (6)). By deleting an edge incident to the starting vertex $s \in C_{0}$ from $H_{0,1}^{\prime \prime}$, we obtain the first alternating Hamiltonian path $P_{0,1}^{\prime}$. Note that the edge is incident to a vertex $v \in C_{1}$, and we must start the second alternating Hamiltonian path $P_{1,2}^{\prime}$ from the vertex $v$ due to the constraint of consecutiveness. Next, we delete such an edge incident to $v \in C_{1}$ from $H_{1,2}^{\prime \prime}$ to obtain $P_{1,2}^{\prime}$. For the remaining transitions, $i=3,4, \ldots, m$, we delete an edge from $H_{i-1, i}^{\prime \prime}$ in a similar manner to obtain the $i$-th alternating Hamiltonian path $P_{i-1, i}^{\prime}$ satisfying the constraint of consecutiveness. Clearly, it holds $w\left(P_{i-1, i}^{\prime}\right) \leq w\left(H_{i-1, i}^{\prime \prime}\right)$ for each $i=1,2, \ldots, m$.

From Lemma 7 and Eq. (10), we have

$$
\begin{aligned}
w\left(P^{\prime}\right) & =\sum_{i=1}^{m} w\left(P_{i-1, i}^{\prime}\right) \leq \sum_{i=1}^{m} 2 d\left(C_{i-1}, C_{i}\right) \\
& =2 d\left(\mathcal{F}^{\prime}\right) \leq 3 d\left(\mathcal{F}^{*}\right) \leq 3 w\left(P^{*}\right) .
\end{aligned}
$$

Regarding the computational effort, from Lemmas 2 and 7 we see that the universal graph can be constructed in time $O\left(m^{2} n^{7}+m^{3} n^{2}\right)$. While an approximate Hamilton path in it can be computed in $O\left(\mathrm{~m}^{3}\right)$ time, this factor is dominated in the process of constructing the universal graph. Therefore, we obtain the following theorem.

Theorem 10: Problem PRR is 3-approximable in $O\left(m^{2} n^{7}+\right.$ $\left.m^{3} n^{2}\right)$ time.

\section{Concluding Remarks}

In this paper, we considered a repetitive routing problem of a single grasp-and-delivery robot used at an actual assembly line of printed circuit boards. There are $n$ identical pins which support a printed circuit board from underneath to prevent it from overbending. The grasp-anddelivery robot is used to arrange the $n$ pins from their current configuration to the next required configuration by transferring them one by one in a transition. Given $m$ printed circuit boards to be processed, an initial configuration of the pins and a prescribed pin to be grasped first in the initial configuration, the problem asks to find a transfer route of the grasp-and-delivery robot which minimizes the route length over all $m$ transitions. In this paper, we formulated a combinatorial optimization problem of finding a shortest walk in a metric graph, and showed that it is polynomially 3approximable. The proposed approximation algorithm was significantly improved from the previously known factors, eight and six. The 3-factor approximation algorithm comes at a cost of higher computational complexity than the previously known algorithms, needed to compute the weights in the universal graph.

The following directions are still open for future research. It would be interesting to investigate a tight instance with the constant factor performance guarantee. It would also be interesting to examine the empirical performance of the proposed approximation algorithm.

\section{Acknowledgment}

This research was partially supported by a Grant-in-Aid for Scientific Research (C) of the Japan Society for the Promotion of Science (JSPS).

\section{References}

[1] A. Baltz, and A. Srivastav, "Approximation algorithms for the Euclidean bipartite TSP," Operations Research Letters, vol.33, pp.403410, 2005.

[2] N. Christofides, "Worst-case analysis of a new heuristic for the travelling salesman problem," Technical Report 388 (Graduate School of Industrial Administration, Carnegie-Mellon University, Pittsburgh, 1976.

[3] J. Edmonds, "Paths, trees, and flowers," Canadian Journal of Mathematics, vol.17, pp.449-467, 1965.

[4] A. Frank, "A weighted matroid intersection algorithm," J. Algorithms, vol.2, pp.328-336, 1981.

[5] M.L. Fredman, and R.E. Tarjan, "Fibonacci heaps and their uses in improved network optimization problems," J. ACM, vol.34, pp.596615, 1987.

[6] M.R. Garey, and D.S. Johnson, Computers and Intractability: A Guide to the Theory of NP-Completeness, W.H. Freeman, San Francisco, 1979.

[7] J.A. Hoogeveen, "Analysis of Christofides' heuristic: Some paths are more difficult than cycles," Oper. Res. Lett, vol.10, pp.291-295, 1991.

[8] D.S. Johnson and C.H. Papadimitriou, "Performance guarantees for heuristics," in The Traveling Salesman Problem: A. Guided Tour of Combinatorial Optimization, E.L. Lawler, J.K. Lenstra, A.H.G. Rinnooy Kan, and D.B. Shmoys, eds., Wiley, Chichester, pp.145180, 1985.

[9] Y. Karuno, H. Nagamochi, and A. Shurbevski, "An approximation algorithm with factor two for a repetitive routing problem of graspand-delivery robots," J. Advanced Computational Intelligence and Intelligent Informatics, vol.15, pp.1103-1108, 2011.

[10] Y. Karuno, H. Nagamochi, and A. Shurbevski, "Approximating cyclic routing problems of grasp-and-delivery robots in production of printed circuit boards," Proc. International Symposium on Scheduling 2011 (ISS 2011), pp.247-252, 2011.

[11] Y. Karuno, H. Nagamochi, and A. Shurbevski, "Constant factor approximation algorithms for repetitive routing problems of graspand-delivery robots in production of printed circuit boards," J. Operations Research Society of Japan, vol.55, pp.181-191, 2012.

[12] B. Korte, and J. Vygen, Combinatorial Optimization: Theory and Algorithms, 3rd Edition, Springer, Berlin, 2005, the Japanese language translation by T. Asano, T. Hirata, T. Ono, and Y. Asano, Springer Tokyo, Tokyo, 2005.

[13] M.S. Krishnamoorthy, "An NP-hard problem in bipartite graphs," SIGACT News, vol.7, p.26, 1975.

[14] R.C. Prim, "Shortest connection networks and some generalization," Bell Syst. Tech. J., vol.36, pp.1389-1401, 1957.

[15] A. Srivastav, H. Schroeter, and C. Michel, "Approximation algorithms for pick-and-place robots," Annals of Operations Research, 


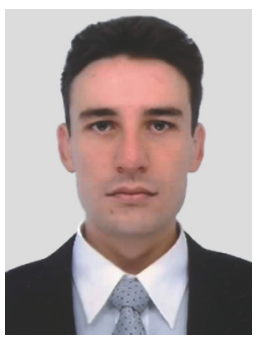

\begin{abstract}
Aleksandar Shurbevski was born in Skopje, Macedonia, on January 13, 1986. He received the B.Sc. degree from the University of St. Cyril and Methodius in 2008, and the M.E. degree from Kyoto Institute of Technology in 2012. As of April 2012, he is a student of the Doctoral Program in the Department of Applied Mathematics and Physics, Graduate School of Informatics, Kyoto University. His research interests include routing and scheduling problems.
\end{abstract}

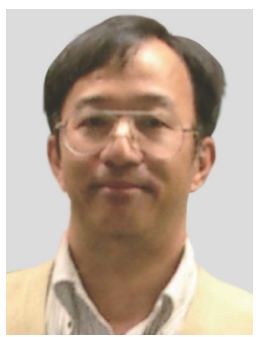

Hiroshi Nagamochi was born in Tokyo, on January 1, 1960. He received the B.A., M.E. and D.E. degrees from Kyoto University, in 1983, in 1985 and in 1988, respectively. He is a Professor in the Department of Applied Mathematics and Physics, Graduate School of Informatics, Kyoto University. His research interests include network flow problems and graph connectivity problems. Dr. Nagamochi is a member of the Operations Research Society of Japan, the Information Processing Society, and the Japan Society for Industrial and Applied Mathematics.

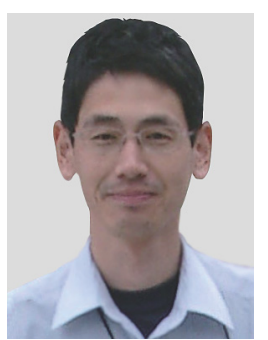

Yoshiyuki Karuno was born on June 5, 1966. He received the M.E. degree and Ph.D. from Kyoto Institute of Technology and Kyoto University, in 1992 and in 2000, respectively. $\mathrm{He}$ is an Associate Professor in the Department of Mechanical and System Engineering, Graduate School of Science and Technology, Kyoto Institute of Technology. His research interests include vehicle scheduling problems and food packing problems. Dr. Karuno is a member of the Operations Research Society of Japan, the Japan Society of Mechanical Engineers, and Scheduling Society of Japan. 